\title{
The myelin oligodendrocyte glycoprotein directly binds nerve growth factor to modulate central axon circuitry
}

\author{
H.-Christian von Büdingen,' Feng Mei, ${ }^{1,2}$ Ariele Greenfield,' Sarah Jahn, ${ }^{1}$ Yun-An A. Shen, ${ }^{1}$ Hugh H. Reid, ${ }^{3}$ \\ David D. McKemy, ${ }^{4}$ and Jonah R. Chan ${ }^{1}$ \\ 'Department of Neurology, University of California, San Francisco, San Francisco, CA 94158 \\ 2Department of Histology and Embryology, Chongaing Key Laboratory of Neurobiology, Third Military Medical University, Chongaing 400038 , China \\ ${ }^{3}$ The Department of Biochemistry and Molecular Biology, School of Biomedical Sciences, Monash University, Clayton, Victoria 3800, Australia \\ 4 Department of Biological Sciences, Neurobiology Section, University of Southern California, Los Angeles, CA 90089
}

Myelin oligodendrocyte glycoprotein (MOG) is a central nervous system myelin-specific molecule expressed on the outer lamellae of myelin. To date, the exact function of MOG has remained unknown, with MOG knockout mice displaying normal myelin ultrastructure and no apparent specific phenotype. In this paper, we identify nerve growth factor (NGF) as a binding partner for MOG and demonstrate that this interaction is capable of sequestering NGF from TrkA-expressing neurons to modulate axon growth and survival. Deletion of MOG results in aberrant sprouting of nociceptive neurons in the spinal cord. Binding of NGF to MOG may offer widespread implications into mechanisms that underlie pain pathways.

\section{Introduction}

Local levels of neurotrophins are thought to promote growth, survival, and stabilization of primary afferents. Levels of brain-derived neurotrophic factor (BDNF) and NT-3 are modulated by truncated forms of TrkB and TrkC (Liu et al., 2012); however, a similar mechanism controlling NGF concentration is not currently identified. Small-diameter nociceptive axons are typically unmyelinated and express TrkA, the high-affinity receptor for NGF. These NGF-dependent axon tracts are surrounded by myelinated fibers in the spinal cord. We therefore hypothesized that myelin itself might modulate central axon outgrowth and circuitry by regulating local concentrations of NGF in the spinal cord microenvironment. Consistent with this hypothesis, recent findings suggest that the ablation of oligodendrocytes and consequent myelin loss in adult mice contributes to axonal pathology and nociceptive hypersensitivity (Gritsch et al., 2014). Therefore, we asked whether myelin itself, or rather, a specific factor expressed by myelin, might modulate central axon outgrowth and circuitry by regulating local concentrations of NGF in the spinal cord microenvironment.

Identification of a binding partner for NGF on myelin necessitates a molecule located on the myelin surface that is readily accessible for binding. Although the major protein components of central nervous system (CNS) myelin consist of myelin basic protein (MBP), myelin associated glycoprotein, and

Correspondence to Jonah R. Chan: jonah.chan@ucsf.edu; or H.-Christian von Büdingen: hans-christian.vonbuedingen@ucsf.edu

Abbreviations used in this paper: BDNF, brain-derived neurotrophic factor; CGRP, calcitonin gene-related peptide; CNS, central nervous system; DRG, dorsal root ganglion; MBP, myelin basic protein; MOG, myelin oligodendrocyte glycoprotein; MS, multiple sclerosis; OPC, oligodendrocyte precursor cell. the proteolipid protein, myelin oligodendrocyte glycoprotein (MOG), a minor component of myelin, is the only protein exclusively restricted to the outermost lamellae of compact CNS myelin (Brunner et al., 1989). In order for a myelin protein to display binding capacity, we further hypothesized that it must share structural features with TrkA. The NGF-binding domain of TrkA is an Ig C2-type domain (Wiesmann et al., 1999). Upon examination of myelin proteins with known Ig domains, two proteins stand out. The first is myelin-associated glycoprotein, which contains V-type and C-type Ig domains but localizes to the periaxonal surface of myelin, making it inaccessible for binding of interstitial soluble factors (Trapp et al., 1989). The second protein is MOG with a V-type Ig extracellular domain (Breithaupt et al., 2003; Clements et al., 2003). Interestingly, the function of MOG remains uncertain as MOG knockout mice do not present any known obvious abnormalities and display normal compact myelin (Delarasse et al., 2003; Liñares et al., 2003). To date, MOG is mainly studied as a highly encephalitogenic antigen in animal models of multiple sclerosis (MS) and as a controversially discussed target antigen of autoimmune responses in human MS (Reindl et al., 2013). Here, we study the direct interaction of NGF with MOG and based on our findings, propose a role for MOG as a novel and specific binding partner for NGF that may modulate local concentrations of the neurotrophin in the spinal cord microenvironment.

(C) 2015 von Büdingen et al. This article is distributed under the terms of an AttributionNoncommercial-Share Alike-No Mirror Sites license for the first six months after the publication date (see http://www.rupress.org/terms). After six months it is available under a Creative Commons License (Attribution-Noncommercial-Share Alike 3.0 Unported license, as described at http://creativecommons.org/licenses/by-nc-sa/3.0/). 


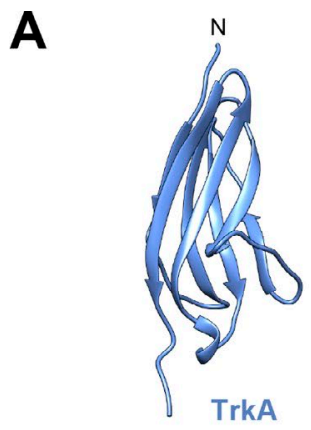

B

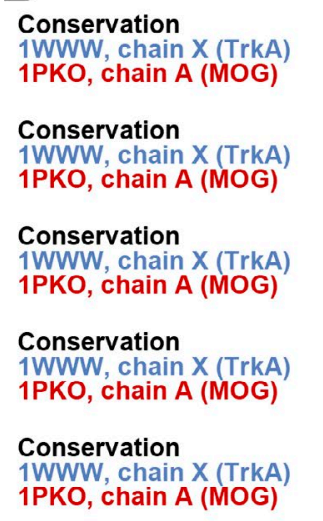

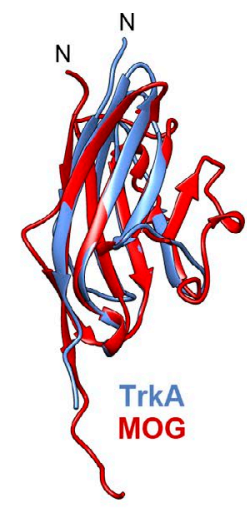
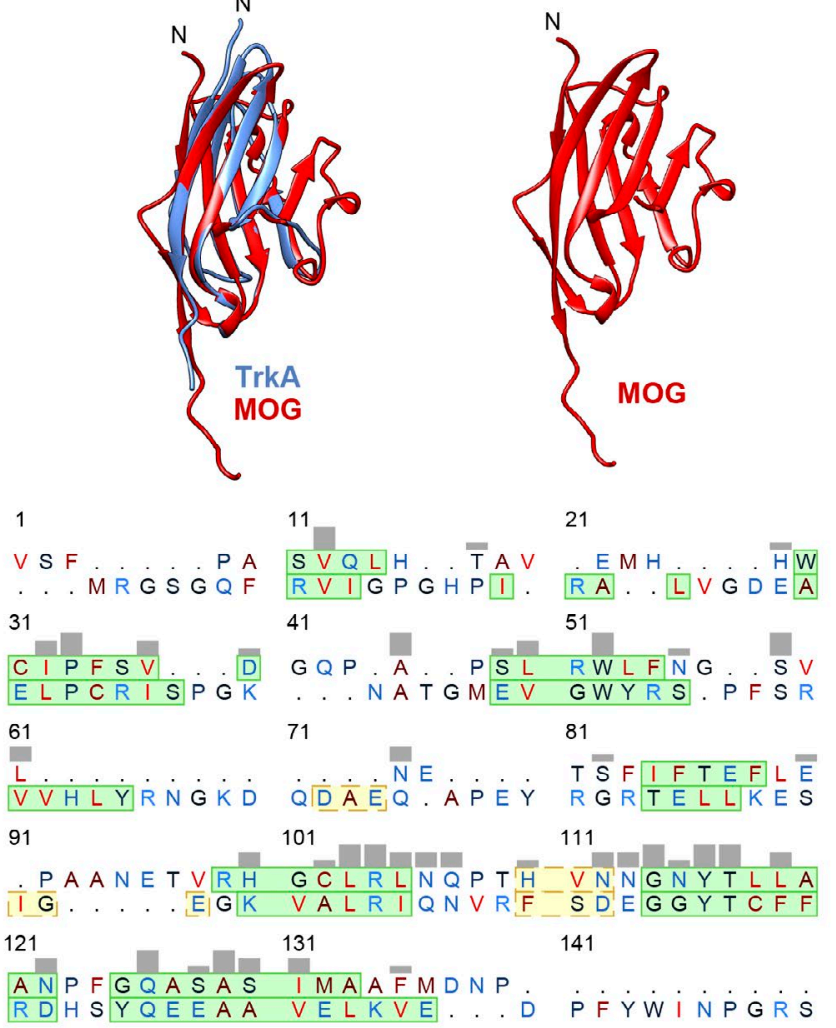

Figure 1. MOG is a novel binding partner for NGF. Structure and sequence alignment were performed in Chimera (Pettersen et al., 2004) using RCSB Protein Data Bank files IWWW (TrkA; Wiesmann et al., 1999) and 1PKO (MOG; Breithaupt et al., 2003). (A) Individual 3D structures of TrkA (1WWW, chain X) and MOG (1PKO, chain A), and their overlay as generated by MatchMaker (Meng et al., 2006) included in Chimera. Both the extracellular domain of MOG and domain 5 of TrkA are lg domains, hence the high degree of structural similarity. (B) Structure-based alignment of IWWA chain X (TrkA, NGF binding domain) and IPKO chain A (MOG). Shown are Clustal Conservation bars atop the sequences. Secondary structure features indicated are strands (green shading) and helices (yellow shading); the hexa-histidine tag of MOG is not shown. (C) Affinity pull-down of MOG-Fc with increasing concentrations of purified neurotrophins $(0-2.0 \mathrm{\mu g} / \mathrm{ml})$ show specific interaction between MOG and NGF. (D) NGF ELISA was performed with immobilized MOG-Fc. con, control.
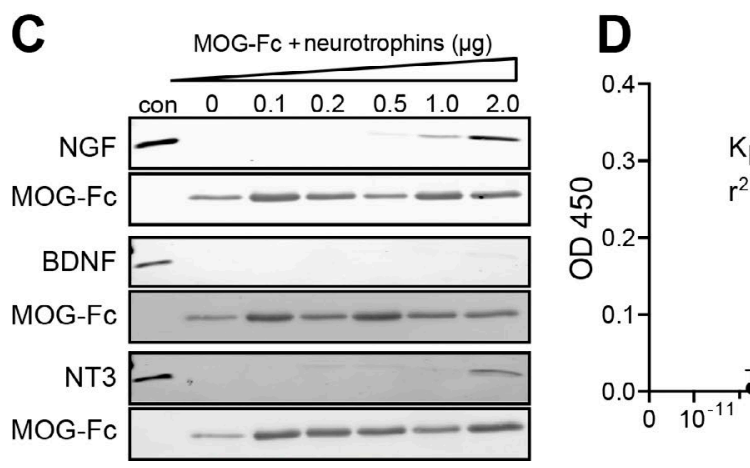

\section{Results and discussion}

MOG is structurally similar to TrkA and is a high-affinity binding partner for NGF

Rudimentary structural comparisons of MOG and TrkA reveal a significant overlap of secondary structures, consistent with the fact that both proteins possess Ig domains (Fig. 1 A). However, little similarity exists between the amino acid sequence of MOG and TrkA (Fig. 1 B). Based on this structural similarity and on the myelin membrane-surface topology of MOG, we focused our efforts on MOG as a potential NGF binding partner that may be capable of regulating NGF availability to TrkA-expressing neurons in the spinal cord, similar to truncated forms of receptors for other neurotrophins.

To gain a basic understanding of NGF binding to MOG, we performed an affinity pull-down assay with recombinant MOG-Fc protein (chimeric protein consisting of the extracellular domain of MOG amino acids 1-125 and human IgG1 Fc) with increasing concentrations of NGF, BDNF, and NT-3 (Fig. $1 \mathrm{C}$ ). MOG specifically pulls down NGF illustrating direct association and binding in vitro. Furthermore, to quan- tify the binding of NGF to MOG, we performed ELISA using the recombinant MOG-Fc protein and determined a binding constant of $<1 \mathrm{nM}$ (Fig. $1 \mathrm{D})$.

\section{NGF binds to myelin and native surface- exposed MOG}

To examine the possible interaction of NGF with myelin, we first established purified oligodendrocyte-neuron co-cultures to recapitulate developmental myelination (Chong et al., 2012). Upon incubation of NGF with myelinated co-cultures, we observe binding of NGF directly to mature myelin internodes (Fig. 2 A). Mature oligodendrocytes and myelin do not express the p75 neurotrophin receptor or full-length TrkA during development or in co-cultures (Chan et al., 2004), suggesting that a different factor must be responsible for this finding. Incubation of NGF with myelinated co-cultures from MOG-deficient oligodendrocytes reveals the lack of binding to myelin internodes, further supporting that MOG is indeed a necessary component for NGF sequestration (Fig. 2 B). To determine whether cell surface-expressed MOG is sufficient for NGF binding, and in an attempt to eliminate the possibility of myelin-expressed 
coreceptors for NGF, we set out to investigate the potential binding of NGF to MOG-expressing $\mathrm{CHO}$ cells. MOG-CHO cells were incubated with increasing concentrations (1-100 ng/ $\mathrm{ml}$ ) of NGF, revealing strong surface binding of NGF at $10 \mathrm{ng} /$ $\mathrm{ml}$ (Fig. $2 \mathrm{C}$ ); no NGF-binding to untransfected $\mathrm{CHO}$ cells was observed (Fig. 2 D). Much weaker binding of NT-3 and an absence of binding of BDNF to MOG-CHO cells were observed at a 10-fold higher concentration than NGF (Fig. 2, E and F), whereas both neurotrophins share structural and sequence similarity with NGF. Collectively, these findings are the first indication that MOG may indeed represent a novel and specific binding partner for NGF expressed on the surface of myelin.

A recent study proposes that ablation of oligodendrocytes in adult mice contributes to axonal pathology and nociceptive hypersensitivity that resembles central neuropathic pain (Gritsch et al., 2014). However, pain tracts are mainly composed of slow-conducting, unmyelinated c-fibers, and a mechanistic connection between pain and oligodendrocyte ablation is lacking. Accordingly, the seemingly paradoxical effect of demyelination on unmyelinated pain fibers necessitates further explanation. In this work, we address the hypothesis that MOG expressed on CNS myelin may exert an indirect effect on the physiological function of unmyelinated NGF-dependent nociceptive pathways. In humans, numerous conditions are known to cause neuropathic pain, including spinal cord injury and MS. Both pathological conditions also coincide with more or less widespread spinal cord demyelination. Importantly, both conditions also present with varying degrees of inflammation as a result of which microglia and astrocytes may be activated to produce NGF (Brown et al., 2004). It is known that in adult animals, increased local levels of NGF in the spinal cord lead to aberrant sprouting of TrkA-expressing nociceptive fibers and contribute to the establishment of chronic neuropathic pain syndromes (Pezet and McMahon, 2006). We thus propose that increased local concentrations of NGF in context with demyelination may result in a disruption of the control mechanism provided by the MOG-NGF interaction, leading to TrkA activation and aberrant sprouting of nociceptive axons.

MOG depletes NGF from the local

environment in vitro

Binding of NGF to MOG suggests an indirect effect of myelin on TrkA-expressing axons. To further understand whether MOG is indeed capable of controlling local NGF concentrations in a given microenvironment, we next examined survival of NGF-dependent dorsal root ganglion (DRG) neurons in the presence or absence of MOG. MOG-expressing $\mathrm{CHO}$ cells were plated into permeable transwell chambers and co-cultured with DRG neurons at varying concentrations of NGF (Fig. 3 A). As DRG neurons are dependent on NGF for survival, we analyzed neurons for apoptosis after $2 \mathrm{~d}$ in culture (Fig. $3 \mathrm{~B}$ ). In the presence of MOG-expressing $\mathrm{CHO}$ cells, a significant increase in apoptotic neurons was observed as compared with control $\mathrm{CHO}$ cells (Fig. 3, A and B), suggesting NGF depletion by MOG-expressing $\mathrm{CHO}$ cells. It is important to note that the control $\mathrm{CHO}$ cells also increase the number of apoptotic neurons at lower concentrations of NGF-albeit to a lesser extent than the MOG$\mathrm{CHO}$ cells. We speculate that this result is caused by the high metabolic activity of $\mathrm{CHO}$ cells and the nonspecific binding of NGF. Nevertheless, these experiments support our hypothesis in that MOG expression is capable of depleting NGF levels in the local environment. To determine whether MOG-expressing
$\mathrm{CHO}$ cells are in fact directly binding and depleting NGF from the culture medium, we coimmunoprecipitated MOG after addition of NGF and chemical cross-linking (Fig. $3 \mathrm{C}$ ). As the molecular mass of MOG is $\sim 25 \mathrm{kD}$, we observed an increased shift in the MOG positive band with the addition of NGF. This band was also detected with an anti-NGF antibody suggesting a specific association of NGF to MOG. These bands were not detected when compared with control CHO cells with NGF (Fig. 3 C). These experiments support our hypothesis that MOG is capable of binding and depleting NGF levels in the local environment.

Absence of MOG results in aberrant sprouting of TrkA-expressing fibers in vivo To investigate the functional effect of MOG binding to NGF in vivo, we compared the morphology and trajectory of central axonal processes from NGF-dependent neurons in spinal cords of wild-type and MOG knockout mice. In the latter mice, MOG is ablated throughout development. We hypothesized that NGF-dependent TrkA- and calcitonin gene-related peptide (CGRP)-expressing nociceptive fiber tracts would experience increased NGF levels throughout development, altering the morphology and/or trajectory of the central axons. Consistent with our hypothesis, we find that the posterolateral tracts in the MOG-deficient mice appear disorganized and frequently observe prominent TrkA- and CGRP-positive fibers sprouting into the more central, myelinated areas of the spinal cord (Fig. 4, A-C). We performed Sholl analyses and generated the respective profiles from spinal cord sections from littermates of wildtype and MOG knockout mice at $2 \mathrm{wk}$ postnatal. The extensive sprouting of nociceptive fiber tracts was quantified by plotting the number of intersections against the radial distance from the center of the concentric rings to the dorsal horn (Fig. 4 D). The total number of intersections was significantly increased in the MOG knockout mice when compared with wild-type mice (Fig. 4 E). Although MOG-deficient mice offer some insight into the in vivo function of MOG during normal CNS development, it is important to note that myelination and MOG expression occur after the specification of NGF-dependent neuronal populations, suggesting that a physiological role of NGF binding to MOG becomes relevant only once myelination of the CNS has been completed. Under certain pathological conditions, which coincide with demyelination and increased NGF levels, the proposed mechanisms of NGF sequestration by MOG may fail and contribute to the establishment of neuropathic pain.

The question, however, is whether there is a broader implication of this interaction, one that bears relevance in commonly occurring situations, conditions under which NGF sequestration by MOG is necessary to maintain pain tract homeostasis and/or to prevent the development of neuropathic pain? Although speculative at this point, our finding may bear significance during spinal cord pathology resulting from peripheral nerve injury. Anatomically, peripheral nerves are far less protected than the spinal cord and are thus more easily exposed to traumatic injury. Interestingly, peripheral nerve injury induces remote spinal cord microglia and astrocyte activation (Coyle, 1998; Colburn et al., 1999; Fu et al., 1999; Zhang et al., 2003), a potential source of increased intraspinal NGF concentrations. Given the high-affinity of NGF binding to MOG, elevated concentrations of spinal cord NGF are likely to be sequestered from TrkA by MOG expressed on surrounding intact myelin, an effect that may be further potentiated by the far greater surface of myelin as compared with TrkA-expressing neurons. Thus, 

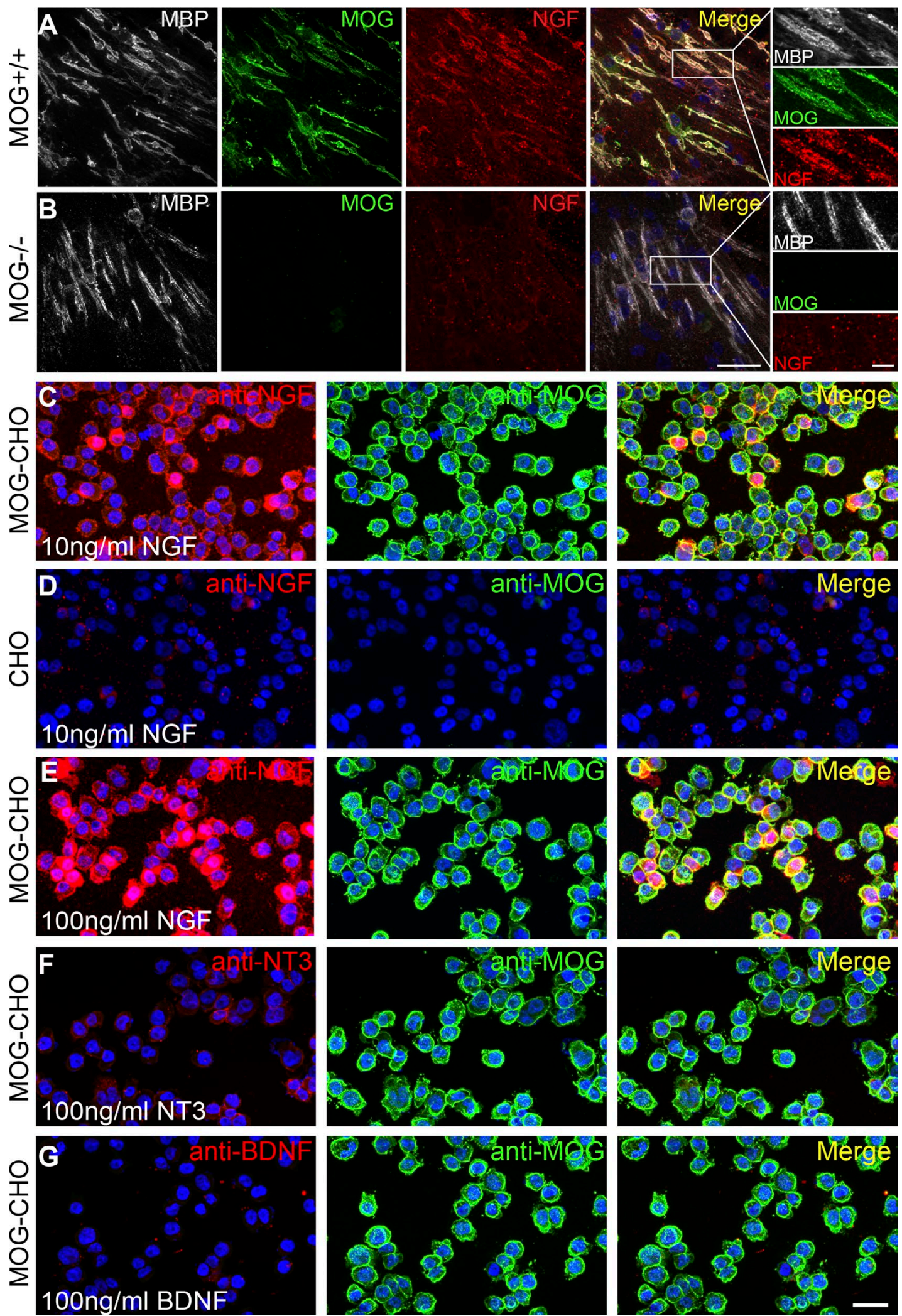

Figure 2. NGF binds to cell surface MOG on myelin and MOG-expressing CHO cells. Purified oligodendrocyte-DRG co-cultures were established using cells from $\mathrm{MOG}^{+/+}$and $\mathrm{MOG}^{-/-}$mice to examine binding of NGF to oligodendrocyte myelin internodes, and requirement of MOG for NGF binding to myelin. Internodes were stained for MBP, MOG, and NGF binding. (A) Binding of NGF to MOG+/+ myelin internodes. (B). Absence of NGF-binding to MOG $-1-$ myelin internodes. Magnified images of internodes from the corresponding merged image are shown on the right. Neurotrophin binding to MOG-expressing $\mathrm{CHO}$ cells was also examined. $\mathrm{CHO}$ or MOG-CHO cells were incubated with increasing concentrations (1-100 ng/ml) of neurotrophins (NGF, NT-3, and BDNF) and analyzed for binding by immunostaining for the respective neurotrophins. (C) Strong of NGF to MOG-CHO cells at $10 \mathrm{ng} /$ $\mathrm{ml} \mathrm{NGF}$. (D) Absence of NGF binding to untransfected CHO cells. (E) Intense binding of NGF at $100 \mathrm{ng} / \mathrm{ml}$ can be observed against MOG-CHO cells. (F and G) Absent or only weak binding of BDNF or NT-3 to MOG-CHO cells at $100 \mathrm{ng} / \mathrm{ml}$. Bars, $50 \mu \mathrm{m}$. 
A
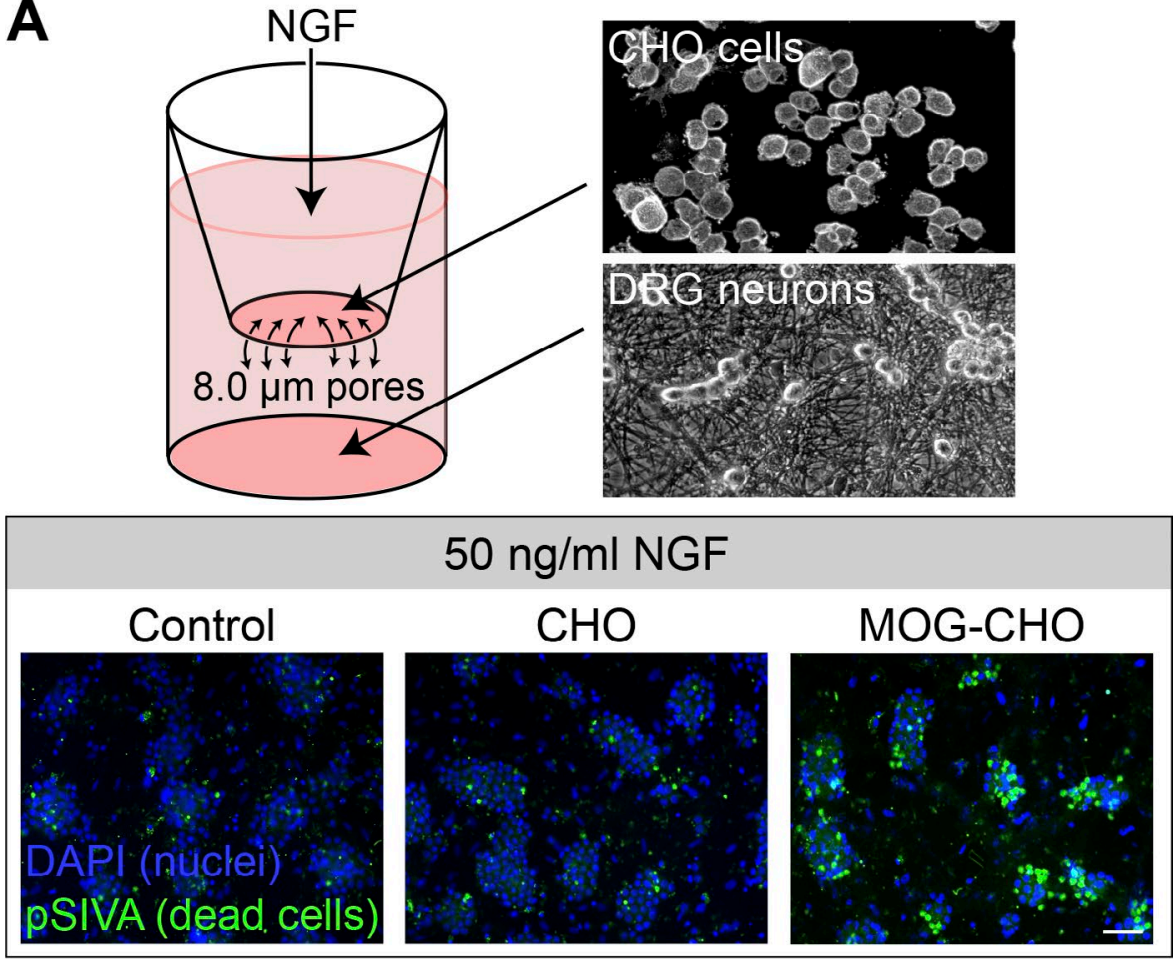

B

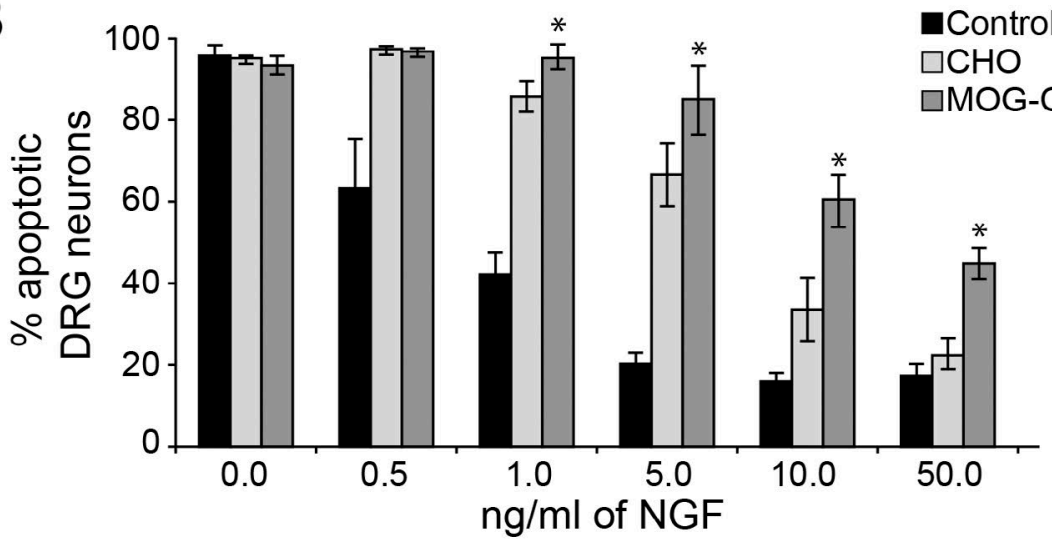

C
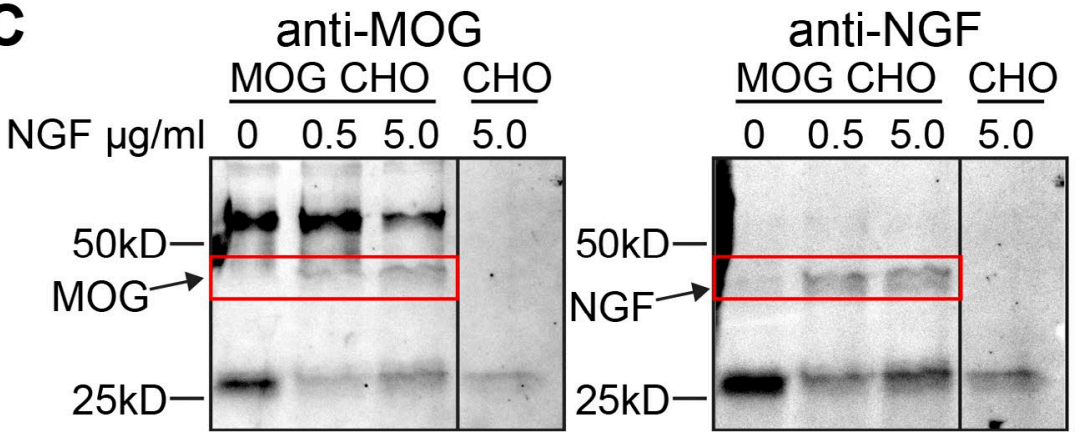

Figure 3. MOG-expressing $\mathrm{CHO}$ cells sequester NGF to control local concentrations of NGF to influence neuronal survival. (A) NGF-dependent dorsal root ganglion (DRG) neurons were plated into permeable transwell chambers and co-cultured with MOG-expressing $\mathrm{CHO}$ cells in the inserts, at varying concentrations of NGF. DRG neurons were analyzed for apoptosis using pSIVA (Kim et al., 2010) after $3 \mathrm{~d}$ in the presence of $\mathrm{CHO}$ cells; shown is the result at $50 \mathrm{ng} / \mathrm{ml}$ NGF. (B) Quantification of DRG survival at different NGF concentrations in the absence of $\mathrm{CHO}$ cells (control), in the presence of the $\mathrm{CHO}$ cells, and in the presence of CHO-MOG cells. Error bars represent SDs, and asterisks represent significance based on Student's $t$ test as compared with the control $\mathrm{CHO}$ cells (*, $\mathrm{P}<0.05)$. (C) Coimmunoprecipitation of $\mathrm{MOG}$ after addition of NGF and chemical cross-linking. MOG-CHO and $\mathrm{CHO}$ cells were incubated with NGF at $500 \mathrm{ng} / \mathrm{ml}$ and $5 \mu \mathrm{g} / \mathrm{ml}$ and chemically crosslinked before coimmunoprecipitation with an anti-MOG antibody. Blots were also probed with an anti-NGF antibody to demonstrate direct association of NGF to MOG.
MOG expression on myelin may have evolved as a protective mechanism to deplete excess NGF and thereby prevent aberrant sprouting and neuropathic pain after peripheral nerve injury. Like many complex biological systems, this protective mechanism may fail at times, and future studies linking CNS demyelination with aberrant sprouting and neuropathic pain may provide valuable insight into the functional consequence for the limited expression of MOG on CNS myelin and NGF binding.
In support of this hypothesis, the neutralization of increased NGF concentrations by intrathecal application of anti-NGF antibodies abolishes sprouting of GCRP-expressing nociceptive neurons in a model of spinal cord injury (Christensen and Hulsebosch, 1997). However, it is also important to note that NGF can up-regulate or modulate the expression of ion channels that are specifically expressed by nociceptors to alter neuronal excitability (Damarjian et al., 2004; Zhang et al., 2005). It 

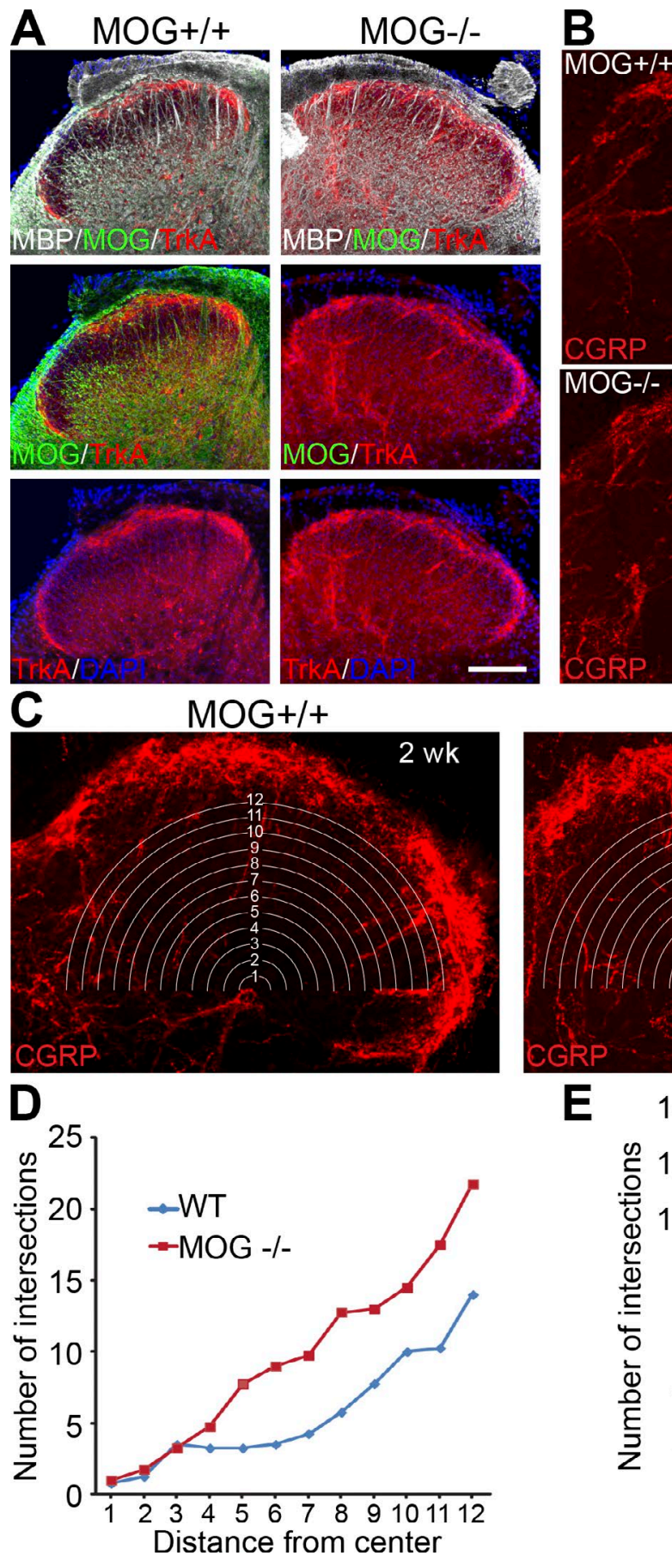

would be interesting to determine whether expression of these ion channels is increased in $\mathrm{MOG}^{-/-}$mice or in other disease states, which could contribute to the hypersensitivity of pain.

In summary, in this study, we not only describe a long sought after function of MOG but also reveal a previously unknown mechanism relevant to the modulation of axon growth and survival of TrkA-expressing axons. We demonstrate the highly specific and affine binding of NGF to MOG and provide evidence that this interaction participates in controlling the histological morphology of the unmyelinated posterolateral tract of the spinal cord. Based on our findings, we conclude that the appearance of MOG on developing mature myelin establishes a regulatory mechanism controlling local concen-
Figure 4. MOG knockout mice display aberrant sprouting of TrkA- and CGRP-positive fibers. (A and B) Pain fiber tracts in spinal cords from wild type (WT; $\mathrm{MOG}^{+/+}$) and $M O G$ knockout $\left(\mathrm{MOG}^{-/-}\right)$were analyzed by staining for TrkA (A) and CGRP (B). Aberrant sprouting of nociceptive axons and increased density of TrkA- and CGRP-positive fibers were observed in $\mathrm{MOG}^{-/-}$compared with $\mathrm{MOG}^{+/+}$mice. (A) Spinal cords from wild-type $\left(\mathrm{MOG}^{+/+}\right)$and MOG knockout $\left(M^{\prime} O G^{-l-}\right.$ ) littermates were analyzed at $14 \mathrm{~d}$ postnatal. Spinal cord sections were matched and immunostained for $M B P, M O G$, and TrkA. (B) Magnified images of CGRP staining from the spinal cord images from 8 wk postnatal mice. (C) Spinal cords from $\mathrm{MOG}^{+/+}$and $\mathrm{MOG}^{-/-}$littermates were analyzed at 2 wk postnatal. Sholl analyses and profiles were generated from spinal cord sections immunostained for CGRP. Bars, 100 um. (D) The number of intersections were plotted against the distance from the center of the concentric circles. The data are represent the mean number of intersections from four spinal cords from the MOG ${ }^{-/-}$mice and four spinal cords from MOG+/+ littermate controls. (E) The total number of intersections was plotted for the wild-type and MOG ${ }^{-/-}$mice. Student's $t$ test was used to compare the total number of intersections in wild-type versus MOG-/- mice $\left({ }^{*}, P<0.05\right)$. Error bars represent SEM. trations of NGF in the vertebrate CNS, comparable to the physiological functions of truncated forms of other neurotrophin receptors (Liu et al., 2012).

\section{Materials and methods}

\section{Oligodendrocyte precursor cell (OPC)-DRGs co-cultures}

OPC-DRG co-cultures were prepared as previously described (Lee et al., 2013). In brief, DRG neurons from embryonic day 15 (E15) Sprague-Dawley rats were dissociated, plated (150,000 cells per 25-mm cover glass), and purified on collagen-coated coverslips in the presence of $100 \mathrm{ng} / \mathrm{ml} \mathrm{NGF} \mathrm{(AbD} \mathrm{Serotec).} \mathrm{Neurons} \mathrm{were} \mathrm{maintained}$ 
for $3 \mathrm{wk}$ and washed with DMEM (Invitrogen) extensively to remove any residual NGF before seeding OPCs. Co-cultures were grown in chemically defined medium composed of DMEM (Invitrogen) supplemented with B27 (Invitrogen), N2 (Invitrogen), penicillin-streptomycin (Invitrogen), $N$-acetyl-cysteine (Sigma-Aldrich), and forskolin (Sigma-Aldrich). To determine the effect of NGF depletion from medium by MOG, $\mathrm{CHO}$, or MOG-expressing $\mathrm{CHO}$ (MOG-CHO) cells (P. Lalive, Geneva University Hospital, Geneva, Switzerland) were co-cultured in permeable transwell inserts.

\section{Immunostaining}

Cultures and spinal cord sections were fixed or perfused, respectively with 4\% PFA, dehydrated, permeabilized, and blocked by incubation with $20 \%$ goat serum (Sigma-Aldrich) and $0.2 \%$ Triton X-100 (Sigma-Aldrich) in PBS as previously described (Chong et al., 2012). Myelin was identified by a rat monoclonal anti-MBP antibody (EMD Millipore) and a mouse monoclonal anti-MOG antibody (EMD Millipore). Nociceptive neurons were detected by a rabbit polyclonal anti-TrkA antibody (gift from L. Reichard, University of California, San Francisco, San Francisco, CA) and a mouse monoclonal anti-CGRP antibody (Abcam). The Alexa Fluor 488, 594, and 647 anti-rat, -rabbit, and -mouse IgG secondary antibodies (1:1,000; Invitrogen) were used for fluorescence detection. Cell nuclei were identified with DAPI (Vector Laboratories). Fluorescent images from co-cultures and spinal cord sections were collected on a fluorescence microscope (Axio Imager Z1; Carl Zeiss) with the excitation wavelengths appropriate for Alexa Fluor 488 (488 nm), 596 (568 nm), 647 (628 nm), or DAPI (380 $\mathrm{nm})$. All images were acquired at room temperature with a Plan Apochromat $20 \times$ objective with a numerical aperture of 0.8 . The sections were mounted in Vectashield mounting medium with DAPI (Vector Laboratories). Images were acquired using a camera (AxioCam MRm; Carl Zeiss). AxioVision software was used for image acquisition, quantification, and analysis.

\section{Affinity pull-down, coimmunoprecipitation, immunoblotting, and Western analysis}

MOG including the MOG-leader sequence was cloned into pFUSEhIgG1-Fc (InvivoGen) as described by others (Zocher et al., 2003) and was expressed as a chimeric homodimer, MOG-Fc, using the Freestyle 293 Expression System (Life Technologies). MOG-Fc and neurotrophins were pulled down in vitro and analyzed by Western blot as previously described (Lewallen et al., 2011). In brief, affinity pull-down of MOG-Fc and varying concentrations of neurotrophins were mixed in buffer containing the following: $50 \mathrm{mM}$ Tris, $\mathrm{pH} 8.0,150 \mathrm{mM} \mathrm{NaCl}$, $2 \mathrm{mM}$ EDTA, $1 \mathrm{mM} \mathrm{Na} \mathrm{VO}_{4}, 2.5 \mathrm{mM} \mathrm{NaF}$, and Complete protease inhibitor (Roche). Protein A/G beads (Santa Cruz Biotechnology, Inc.) were added to the samples and allowed to rotate at $4^{\circ} \mathrm{C}$ overnight. Samples were washed three times in lysis buffer, resuspended in sample buffer with SDS and $\beta$-mercaptoethanol, and boiled for 2 min before loading. Electrophoretic separation of the samples and the extracts was performed on $10 \%$ discontinuous SDS polyacrylamide gels. Proteins were transferred to nitrocellulose membranes and probed with specific antibodies. NGF was identified by a rabbit polyclonal anti-NGF antibody (Sigma-Aldrich), and BDNF was identified by a chicken polyclonal anti-BDNF antibody (Promega) and a chicken polyclonal anti-NT-3 antibody (Promega). The Alexa Fluor goat anti-rabbit, antimouse, and anti-chicken $680 \mathrm{IgG}$ antibodies (Invitrogen) were used as secondary antibodies for near-infrared fluorescent detection performed on the Odyssey Infrared Imaging System (LI-COR Biosciences). Coimmunoprecipitation of full-length MOG from the MOG-CHO cells at varying concentrations of NGF was performed after chemical cross-linking using a water-soluble analogue of $N$-hydroxysuccinimide ester, BS $^{3}$
(Thermo Fisher Scientific). In brief, MOG-CHO cells and control CHO cells were incubated with NGF at $500 \mathrm{ng}$ and $5 \mu \mathrm{g}$ for $1 \mathrm{~h}$ at room temperature. Cells were washed and cross-linked with $5 \mathrm{mM} \mathrm{BS}^{3}$ for $1 \mathrm{~h}$ at room temperature. The reaction was quenched with $15 \mathrm{mM}$ Tris buffer for $15 \mathrm{~min}$ at room temperature. Cells were then homogenized in buffer containing: $50 \mathrm{mM}$ Tris, $\mathrm{pH}$ 8.0, $150 \mathrm{mM} \mathrm{NaCl}, 2 \mathrm{mM}$ EDTA, $1 \mathrm{mM}$ $\mathrm{Na}_{3} \mathrm{VO}_{4}, 2.5 \mathrm{mM} \mathrm{NaF}$, and Complete protease inhibitor (Roche). Protein $\mathrm{A} / \mathrm{G}$ beads and a mouse anti-MOG antibody $(818 \mathrm{C} 5)$ were added to the samples and allowed to rotate at $4^{\circ} \mathrm{C}$ overnight. Samples were washed three times in lysis buffer, resuspended in sample buffer with SDS and $\beta$-mercaptoethanol, and boiled for $2 \mathrm{~min}$ before Western analysis. MOG protein was probed using the human anti-MOG antibody (818C5), and NGF was probed using the rabbit anti-NGF antibody (Sigma-Aldrich).

\section{ELISA}

Binding of NGF to MOG was studied by ELISA. In brief, the MOG-Fc fusion protein was used as the capturing reagent followed by incubation with samples containing increasing concentrations of NGF. Bound NGF was detected using a polyclonal rabbit anti-NGF primary antibody (Sigma-Aldrich) followed with an anti-rabbit HRP antibody (Promega). To measure the depletion of NGF by MOG-CHO, CHO, or MOG-CHO were cultured in medium containing increasing concentrations of NGF. NGF concentrations in supernatant were measured after 3-h incubation by a sandwich ELISA as described previously ( $\mathrm{Ng}$ et al., 2007). In brief, ELISA wells were coated with an anti-NGF capture antibody (Abcam) followed by incubation with samples. Bound NGF was detected using a biotinylated anti-NGF antibody (Abcam) followed by incubation with streptavidin-HRP (Abcam). All ELISAs were performed using tetramethylbenzidine (Life Technologies) as an HRP substrate. The substrate was incubated for $2-5 \mathrm{~min}$ or until adequate signal was detected. ELISA reactions were stopped with the addition of $1 \mathrm{M}$ sulfuric acid.

\section{ACKNOWLEDGMENTS}

We thank the Chan and von Büdingen laboratories, Dr. Stephen Hauser for support, advice, and insightful discussions, and Dr. Patrice Lalive for the MOG-CHO cells.

This work was supported by the National Multiple Sclerosis Society Harry Weaver Neuroscience Scholar Award (JF2 142-A-2), the National Institutes of Health/National Institute of Neurological Disorders and Stroke (NINDS; R01NS062796) to J.R. Chan, National Institutes of Health/NINDS (K02NS072288) to H.-C. von Büdingen, and the Rachleff Professorships to J.R. Chan and H.-C. von Büdingen.

The authors declare no competing financial interests.

Submitted: 23 April 2015

Accepted: 29 July 2015

\section{References}

Breithaupt, C., A. Schubart, H. Zander, A. Skerra, R. Huber, C. Linington, and U. Jacob. 2003. Structural insights into the antigenicity of myelin oligodendrocyte glycoprotein. Proc. Natl. Acad. Sci. USA. 100:9446-9451. http://dx.doi.org/10.1073/pnas.1133443100

Brown, A., M.J. Ricci, and L.C. Weaver. 2004. NGF message and protein distribution in the injured rat spinal cord. Exp. Neurol. 188:115-127. http:// dx.doi.org/10.1016/j.expneurol.2004.03.017

Brunner, C., H. Lassmann, T.V. Waehneldt, J.M. Matthieu, and C. Linington. 1989. Differential ultrastructural localization of myelin basic protein, myelin/oligodendroglial glycoprotein, and 2',3'-cyclic nucleotide $3^{\prime}$-phos- 
phodiesterase in the CNS of adult rats. J. Neurochem. 52:296-304. http:// dx.doi.org/10.1111/j.1471-4159.1989.tb10930.x

Chan, J.R., T.A. Watkins, J.M. Cosgaya, C. Zhang, L. Chen, L.F. Reichardt, E.M. Shooter, and B.A. Barres. 2004. NGF controls axonal receptivity to myelination by Schwann cells or oligodendrocytes. Neuron. 43:183-191. http://dx.doi.org/10.1016/j.neuron.2004.06.024

Chong, S.Y., S.S. Rosenberg, S.P. Fancy, C. Zhao, Y.A. Shen, A.T. Hahn, A.W. McGee, X. Xu, B. Zheng, L.I. Zhang, et al. 2012. Neurite outgrowth inhibitor Nogo-A establishes spatial segregation and extent of oligodendrocyte myelination. Proc. Natl. Acad. Sci. USA. 109:1299-1304. http:// dx.doi.org/10.1073/pnas.1113540109

Christensen, M.D., and C.E. Hulsebosch. 1997. Spinal cord injury and anti-NGF treatment results in changes in CGRP density and distribution in the dorsal horn in the rat. Exp. Neurol. 147:463-475. http://dx.doi.org/10.1006/ exnr.1997.6608

Clements, C.S., H.H. Reid, T. Beddoe, F.E. Tynan, M.A. Perugini, T.G. Johns, C.C. Bernard, and J. Rossjohn. 2003. The crystal structure of myelin oligodendrocyte glycoprotein, a key autoantigen in multiple sclerosis. Proc. Natl. Acad. Sci. USA. 100:11059-11064. http://dx.doi.org/10.1073/ pnas. 1833158100

Colburn, R.W., A.J. Rickman, and J.A. DeLeo. 1999. The effect of site and type of nerve injury on spinal glial activation and neuropathic pain behavior. Exp. Neurol. 157:289-304. http://dx.doi.org/10.1006/exnr.1999.7065

Coyle, D.E. 1998. Partial peripheral nerve injury leads to activation of astroglia and microglia which parallels the development of allodynic behavior. Glia. 23:75-83. http://dx.doi.org/10.1002/ (SICI) 1098-1136(199805)23:1<75::AID-GLIA7>3.0.CO;2-3

Damarjian, T.G., M.J. Craner, J.A. Black, and S.G. Waxman. 2004. Upregulation and colocalization of p75 and Nav1.8 in Purkinje neurons in experimental autoimmune encephalomyelitis. Neurosci. Lett. 369:186-190. http:// dx.doi.org/10.1016/j.neulet.2004.07.023

Delarasse, C., P. Daubas, L.T. Mars, C. Vizler, T. Litzenburger, A. Iglesias, J. Bauer, B. Della Gaspera, A. Schubart, L. Decker, et al. 2003. Myelin/ oligodendrocyte glycoprotein-deficient (MOG-deficient) mice reveal lack of immune tolerance to MOG in wild-type mice. J. Clin. Invest. 112:544553. http://dx.doi.org/10.1172/JCI15861

Fu, K.Y., A.R. Light, G.K. Matsushima, and W. Maixner. 1999. Microglial reactions after subcutaneous formalin injection into the rat hind paw. Brain Res. 825:59-67. http://dx.doi.org/10.1016/S0006-8993(99)01186-5

Gritsch, S., J. Lu, S. Thilemann, S. Wörtge, W. Möbius, J. Bruttger, K. Karram, T. Ruhwedel, M. Blanfeld, D. Vardeh, et al. 2014. Oligodendrocyte ablation triggers central pain independently of innate or adaptive immune responses in mice. Nat. Commun. 5:5472. http://dx.doi.org/10.1038/ ncomms6472

Kim, Y.E., J. Chen, R. Langen, and J.R. Chan. 2010. Monitoring apoptosis and neuronal degeneration by real-time detection of phosphatidylserine externalization using a polarity-sensitive indicator of viability and apoptosis. Nat. Protoc. 5:1396-1405. http://dx.doi.org/10.1038/nprot.2010.101

Lee, S., S.Y. Chong, S.J. Tuck, J.M. Corey, and J.R. Chan. 2013. A rapid and reproducible assay for modeling myelination by oligodendrocytes using en- gineered nanofibers. Nat. Protoc. 8:771-782. http://dx.doi.org/10.1038/ nprot.2013.039

Lewallen, K.A., Y.A. Shen, A.R. De la Torre, B.K. Ng, D. Meijer, and J.R. Chan. 2011. Assessing the role of the cadherin/catenin complex at the Schwann cell-axon interface and in the initiation of myelination. J. Neurosci. 31:3032-3043. http://dx.doi.org/10.1523/JNEUROSCI.4345-10.2011

Liñares, D., P. Mañá, M. Goodyear, A.M. Chow, C. Clavarino, N.D. Huntington, L. Barnett, F. Koentgen, R. Tomioka, C.C. Bernard, et al. 2003. The magnitude and encephalogenic potential of autoimmune response to MOG is enhanced in MOG deficient mice. J. Autoimmun. 21:339-351. http:// dx.doi.org/10.1016/j.jaut.2003.09.001

Liu, Y., M. Rutlin, S. Huang, C.A. Barrick, F. Wang, K.R. Jones, L. Tessarollo, and D.D. Ginty. 2012. Sexually dimorphic BDNF signaling directs sensory innervation of the mammary gland. Science. 338:1357-1360. http:// dx.doi.org/10.1126/science. 1228258

Meng, E.C., E.F. Pettersen, G.S. Couch, C.C. Huang, and T.E. Ferrin. 2006. Tools for integrated sequence-structure analysis with UCSF Chimera. BMC Bioinformatics. 7:339. http://dx.doi.org/10.1186/1471-2105-7-339

Ng, B.K., L. Chen, W. Mandemakers, J.M. Cosgaya, and J.R. Chan. 2007. Anterograde transport and secretion of brain-derived neurotrophic factor along sensory axons promote Schwann cell myelination. J. Neurosci. 27:7597-7603. http://dx.doi.org/10.1523/JNEUROSCI.0563-07.2007

Pettersen, E.F., T.D. Goddard, C.C. Huang, G.S. Couch, D.M. Greenblatt, E.C. Meng, and T.E. Ferrin. 2004. UCSF Chimera - a visualization system for exploratory research and analysis. J. Comput. Chem. 25:16051612. http://dx.doi.org/10.1002/jcc. 20084

Pezet, S., and S.B. McMahon. 2006. Neurotrophins: mediators and modulators of pain. Аnпи. Rev. Neurosci. 29:507-538. http://dx.doi.org/10.1146/ annurev.neuro.29.051605.112929

Reindl, M., F. Di Pauli, K. Rostásy, and T. Berger. 2013. The spectrum of MOG autoantibody-associated demyelinating diseases. Nat Rev Neurol. 9:455461. http://dx.doi.org/10.1038/nrneurol.2013.118

Trapp, B.D., S.B. Andrews, C. Cootauco, and R. Quarles. 1989. The myelin-associated glycoprotein is enriched in multivesicular bodies and periaxonal membranes of actively myelinating oligodendrocytes. J. Cell Biol. 109:2417-2426. http://dx.doi.org/10.1083/jcb.109.5.2417

Wiesmann, C., M.H. Ultsch, S.H. Bass, and A.M. de Vos. 1999. Crystal structure of nerve growth factor in complex with the ligand-binding domain of the TrkA receptor. Nature. 401:184-188. http://dx.doi.org/10.1038/43705

Zhang, J., C. Hoffert, H.K. Vu, T. Groblewski, S. Ahmad, and D. O'Donnell. 2003. Induction of CB2 receptor expression in the rat spinal cord of neuropathic but not inflammatory chronic pain models. Eur. J. Neurosci. 17:2750-2754. http://dx.doi.org/10.1046/j.1460-9568.2003.02704.x

Zhang, X., J. Huang, and P.A. McNaughton. 2005. NGF rapidly increases membrane expression of TRPV1 heat-gated ion channels. EMBO J. 24:42114223. http://dx.doi.org/10.1038/sj.emboj.7600893

Zocher, M., P.A. Baeuerle, T. Dreier, and A. Iglesias. 2003. Specific depletion of autoreactive B lymphocytes by a recombinant fusion protein in vitro and in vivo. Int. Immunol. 15:789-796. http://dx.doi.org/10.1093/intimm/ dxg076 